\title{
Embryo to Ashes Complete Evolutionary Tracks, Hands-off
}

\author{
O. Yaron ${ }^{1}$, A. Kovetz ${ }^{1,2}$ and D. Prialnik ${ }^{1}$ \\ ${ }^{1}$ Department of Geophysics and Planetary Sciences, Sackler Faculty of Exact Sciences \\ ${ }^{2}$ School of Physics \& Astronomy, Sackler Faculty of Exact Sciences, Tel Aviv University, Israel
}

\begin{abstract}
We present a new stellar evolution code and a set of results, showing its capability to calculate full evolutionary tracks for a wide range of masses and metalicities. The code is meant to be used also in the context of modeling the evolution of dense stellar systems, for performing live evolutionary calculations both for 'normal' ZAMS/PRE-MS models, but mainly for 'non-canonical' (i.e. merger-products) stellar configurations. For such tasks, it has to be robust and efficient, capable to run through all phases of stellar evolution without interruption or intervention. Here we show a few examples of evolutionary calculations for stellar populations I and II, and for masses in the range $0.25-64 M_{\odot}$.
\end{abstract}

Keywords. stars: evolution, Hertzsprung-Russell diagram

\section{The Evolution Code}

Basic Scheme The equations governing the structure and evolution of a star are those of continuity, hydrostatic equilibrium, energy transfer (radiative or convective), energy balance and composition balance. These differential equations are approximated by finite difference equations, to be solved by an iterative Newton-Raphson method. Following Eggleton (1971), the equations of structure and composition are solved simultaneously with a mass distribution function - implementing an adaptive mesh.

Input Physics Equations of state include Coulomb and quantum corrections; $\mathrm{H}$ and $\mathrm{He}$ ionization equilibria, $\mathrm{H}_{2}$ creation, pair creation and pressure ionization (Pols et al. 1995) are taken into account. Using OPAL opacities (Iglesias \& Rogers 1996) for high temperatures $(4.50<\log T<8.70)$ and Ferguson et al. (2005) for low temperatures, we create for a required metalicity our own set of opacity tables, covering both the Hydrogen mass fractions and Carbon/Oxygen excesses mass fractions from 0 to $[1-Z]$. Electron scattering are incorporated for the highest temperatures according to Iben's fit. The radiative opacities $-\kappa_{\text {rad }}$ are supplemented with conductive opacities $-\kappa_{\text {cond }}$ (when applicable) as obtained from Cassisi et al. (2007). Nuclear reaction rates are from Caughlan \& Fowler (1988) - following H, $\alpha$ and CO - burning. Neutrino losses are according to Itoh et al. (1996) - accounting for neutrino formation processes of pair annihilation, photo annihilation, plasma decay, bremsstrahlung and recombination. Several mass-loss recipes can be tested, variations to the original Reimers (1975) for advanced (POST-RGB) stages. The results displayed in this work were obtained using Bloecker (1995) $\dot{M}_{B 1}$ expression (based on Bowen 1988).

Computational details Our automatically varying time steps, determined mainly by limits imposed on the changes allowed during time step, span a wide dynamic range from $\lesssim 1$ sec (e.g. core He flash) to $\gtrsim 10^{8}$ years (MS). The grid mass shells span a range of $\sim 10^{-15} M_{\odot}$ (WD atmosphere) to $\gtrsim 10^{-1} M_{\odot}$ (inert stellar core). The typical number of grid points is kept in between 150 and 200, and a typical number of time steps for a complete evolution track is 1000 . 
(a)

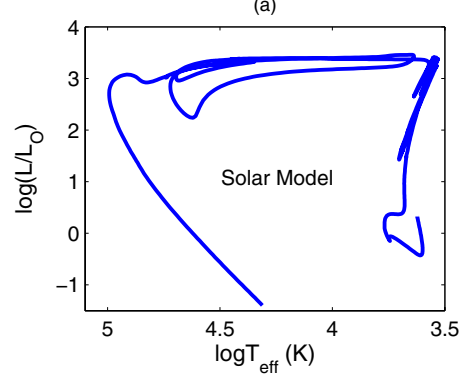

(c)

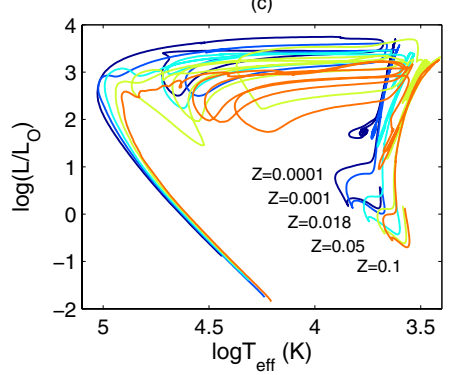

(b)

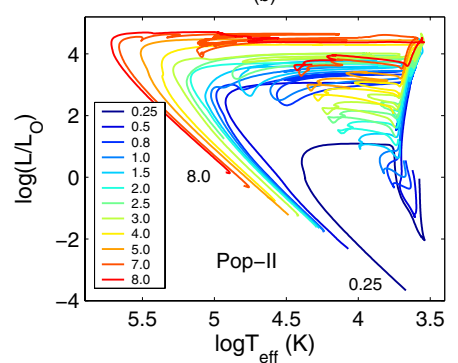

(d)

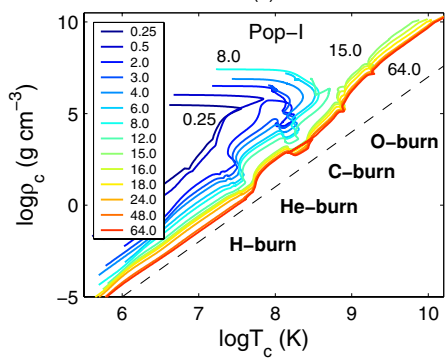

Figure 1. (a) Solar model $-1 M_{\odot}, Y=0.29, Z=0.018$. (b) Complete tracks for Pop. II models $(Z=0.001), 0.25-8.0 M_{\odot}$. (c) Varying metalicities $Z=0.0001$ to 0.1 for $1 M_{\odot}$. MS $T_{\text {eff }}$ and $L$ decrease with increasing Z's; consequently - duration on the MS increases $\left(\tau_{M S}=5.43,5.71,9.01,14.8\right.$ and $18.3 \mathrm{Gyr}$ for the $5 \mathrm{Z}$ 's -0.0001 to 0.1 , respectively). (d) Evolution of the central stellar density and pressure of Pop. I models in the range $0.25-64 M_{\odot}$. Dotted line has a slope of 3 (as obtained for the $\log \rho_{c}-\log P_{c}$ relation of hydrostatic equilibrium under ideal gas law). Nuclear burning phases are marked along the tracks.

\section{Results}

In the panels of Fig. 1 we present selected preliminary calculations (serving mainly for proof of capability, specific applications are to follow). Panel (a) shows a calibration solar model, following through all evolutionary stages and ending as a cooling $0.55 M_{\odot} \mathrm{CO}$ WD. Features at 4.5 Gyr match the present sun to an accuracy of $1 \%$ or better. Panel (d) shows the evolution of the stellar central points in $\log \rho_{c}--\log T_{c}$ plane, exhibiting the branching off between intermediate-mass stars that end their lives as WDs, and massive stars that go through advanced nuclear burning stages, ending their lives in dynamic core collapse (SN) - followed by the code until very high central pressures are attained and the adiabatic exponent approaches $4 / 3$ throughout the core. Future work will extend the study and analysis of non-canonical evolution.

\section{References}

Bloecker, T. $1995 A \& A 297,727$

Bowen, G. H. 1988 ApJ 329, 299

Cassisi, S., Potekhin, A. Y., Pietrinferni, A., Catelan, M., \& Salaris M. 2007 ApJ 661, 1094

Caughlan, G. R. \& Fowler, W. A. 1988 Atomic Data and Nuclear Data Tables 40, 283

Eggleton, P. P. 1971, MNRAS 151, 351

Ferguson, J. W., Alexander, D. R., et al. 2005 ApJ 623, 585

Iglesias, C. A. \& Rogers, F. J. 1996, ApJ 464, 943

Itoh, N., Hayashi, H., Nishikawa, A., \& Kohyama, Y. 1996 ApJS 102, 411

Pols, O. R., Tout, C. A., Eggleton, P. P. \& Han, Z. 1995, MNRAS 274, 964

Reimers, D. 1975 MSRSL 8, 369 